Document downloaded from:

http://hdl.handle.net/10251/100800

This paper must be cited as:

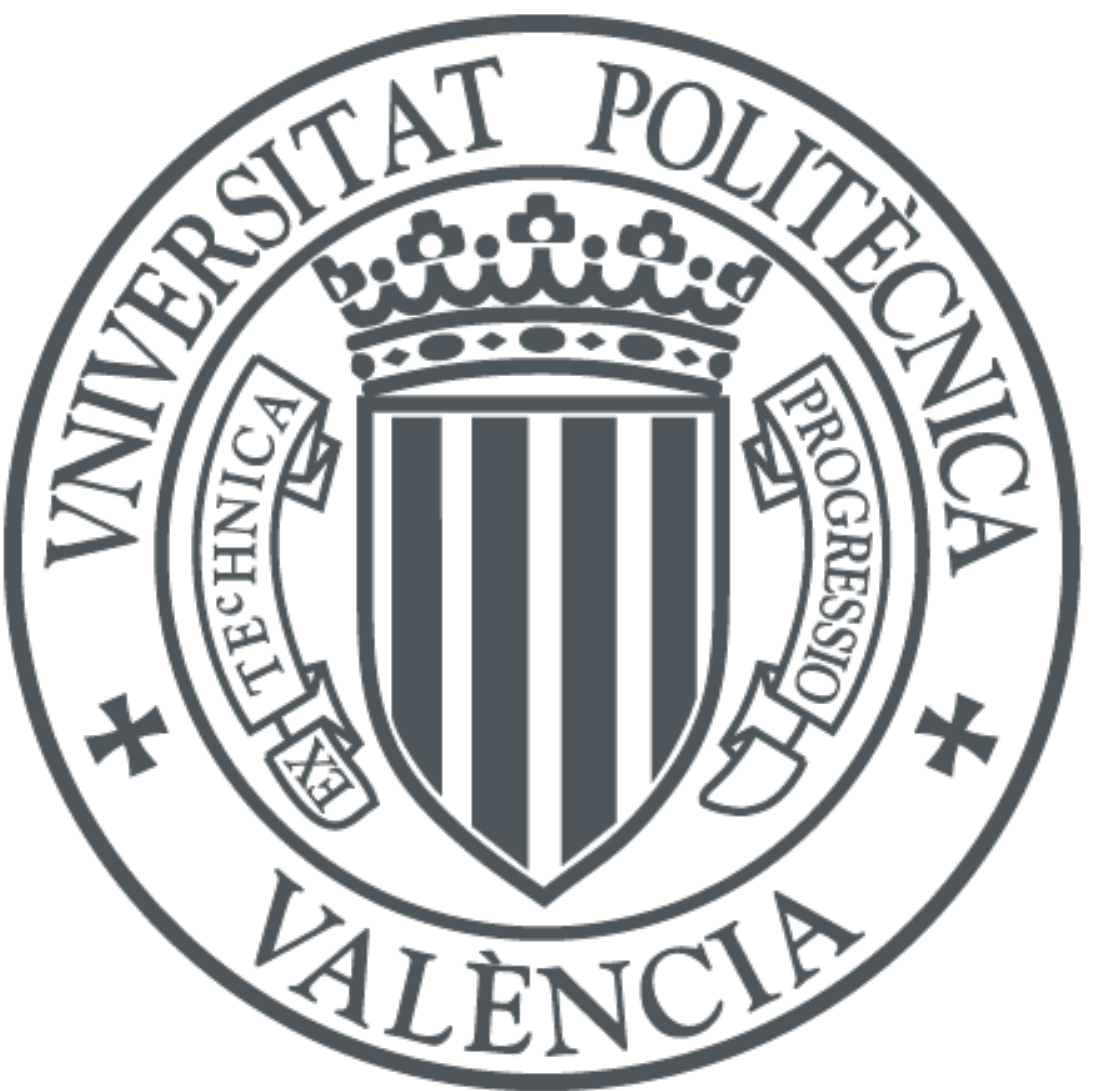

The final publication is available at https://doi.org/10.1109/20.914378

Copyright Institute of Electrical and Electronics Engineers

Additional Information 


\title{
Power Absorption and Ferromagnetic Resonance in Co-Rich Metallic Glasses
}

\author{
H. García-Miquel, M. J. Esbrí, J. M. Andrés, J. M. García, J. M. García-Beneytez, and M. Vázquez, Member, IEEE
}

\begin{abstract}
The power absorption of Co-rich metallic glasses in the shape of ribbons and microwires has been measured in the microwave frequency range. The technique employed consists of replacing the dielectric of a coaxial transmission line by the amorphous sample to be measured. The ferromagnetic resonance frequency of the microwires has been obtained by analyzing the absorbed power as a function of an external magnetic field. From the evolution of this resonance frequency, the anisotropy field has been deduced and successfully compared to that obtained from low-frequency hysteresis loops.
\end{abstract}

Index Terms-Amorphous materials, ferromagnetic resonance, microwave materials.

\section{INTRODUCTION}

$\mathbf{T}$ HE characterization of high-frequency properties of various types of magnetic materials has attracted much attention in the last few years in connection with their technological applications. Particular interest is being focused on peculiar properties such as magnetoimpedance and microwave behavior of extremely soft magnetic materials with metallic character. Such materials, typically amorphous, are fabricated in the shape of wires with diameters in the range from 1 to $150 \mu \mathrm{m}$, ribbons and tubes around $20-\mu \mathrm{m}$ thick, and films in the range of $1 \mu \mathrm{m}$ in thickness [1]-[5]. Although in most cases they are prepared by rapid solidification techniques, electrochemical deposition or sputtering methods are also alternatively employed. These materials have been proposed in applications as active elements in magnetic shielding [6] or to make use of their absorption characteristics.

Several techniques are currently being used to characterize the microwave behavior of different materials. Among them, we can mention the resonant cavity [7], the guided-wave technique [8], measurements in free space [9], the microstrip line [10], using a discontinuity in a coaxial line [11] or replacing the inner conductor [12] or the dielectric [13] by the magnetic material under study.

The aim of this work has been the determination of the power absorption and the ferromagnetic resonance characteristics in

This work was

partially developed under Project MAT/98 0965. J. M. García was supported by a grant from Volkswagen Audi-CSIC.

H. García-Miquel and M. J. Esbrí are with the Departamento Ingenieria Electrónica E.T.S.I.T., Universidad Politécnica de Valencia, 46.022 Valencia, Spain.

J. M. Andrés is with the Laboratorio de Calibración, Universidad Politécnica de Valencia, 46.022 Valencia, Spain.

J. M. García, J. M. García-Beneytez, and M. Vázquez are with the Instituto de Magnetismo Aplicado (RENFE-UCM) and the Instituto de Ciencia de Materiales de Madrid (CSIC), 28230 Las Rozas (Madrid), Spain. metallic glasses such as microwires and melt-spun ribbons using an alternative method. The technique employed here consists of the insertion of the sample into a coaxial line replacing the dielectric between the inner and the outer conductor. The samples are placed in the coaxial line in different arrangements depending on the specific type of measurement. The absorption spectrum of the metallic glasses can be obtained by winding the samples around the inner conductor. In the case of microwires, the ferromagnetic resonance can also be obtained from the absorption measurements by placing the samples parallel to the axis of the coaxial line and applying a field parallel to it.

\section{MeAsurement TechniQues}

\section{A. Samples Used in the Experiments}

Two kinds of metallic glasses have been used in the present study, both of them fabricated in the experimental facilities in our laboratory. Amorphous ribbons with nominal composition $\mathrm{Co} \quad \mathrm{Si} \quad \mathrm{B}$ have been prepared by a conventional melt-spinติīng tealỹnique, being $17 \mathrm{~m}$ thick and $0.6 \mathrm{~mm}$ wide. On the other hand, glass-coated $\mathrm{C} \quad \mathrm{B} \quad \mathrm{Si} \mathrm{Mn}$ amorphous microwires were obtained by the Ta\&olotulifovsky technique, having a total diameter of around $5 \mathrm{~m}$ and a metallic core of $2 \mathrm{~m}$. Recently, these types of tamorphous wires have been ifftensively studied owing to their outstanding magnetic properties. A more detailed description of their characteristics can be found elsewhere [14], [15]. Low-frequency magnetic characteristics of the studied samples have been determined using vibrating sample magnetometry (VSM).

\section{B. Power Absorption}

The technique used to evaluate the power absorption is based on the measurement of the incident, the reflected, and the transmitted power through a modified coaxial transmission line. In this line, the sample is wound around the inner conductor, and replaces the dielectric [16]. A network is thus generated, consisting of three quadrupoles in cascade: the sections of coaxial line of lengths and , and the sample between these sections, as schematically showh in Fig. 1(a).

The scattering parameters which can be measured belong to the whole system. However, the attenuation due to the coaxial lines next to the sample ( and ) is negligible, and causes a displacement in the referende platies that affects only the phase of the scattering parameters but not their modulus. The scattering parameters of the whole system can then be associated with the properties of the sample under study. 


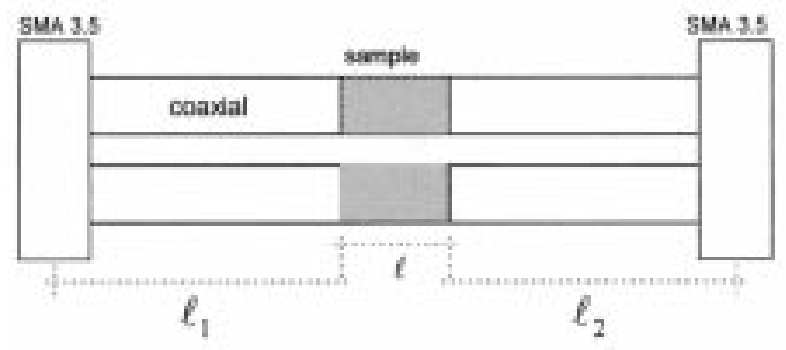

(a)

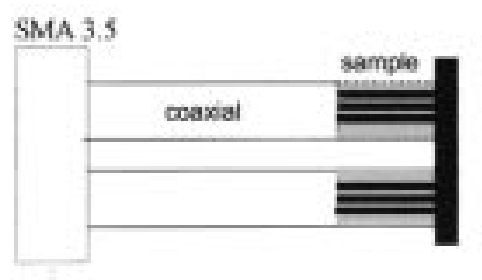

(b)

Fig. 1. Schematic view of the coaxial line arrangements used in the measurements. (a) Arrangement used for the measurement of the power absorption. The dielectric is substituted by the magnetic material. (b) Arrangement for the determination of the resonance frequency.

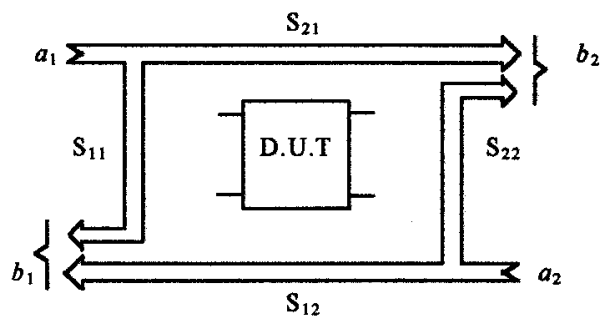

Fig. 2. Scheme for the determination of the scattering parameters (D.U.T. stands for device under test).

The total power absorbed by the system, $\boldsymbol{P}_{\mathbf{a b s}}$ can be normalized with respect to the power available from the source as

$$
P_{\mathbf{a b s}}=1-\left|S_{11}\right|^{2}-\left|S_{21}\right|^{2}
$$

where $S_{11}$ and $S_{21}$ are the scattering parameters of the network. As part of the available power from the source is reflected in the gate port (see Fig. 2), not all the radiation reaches the sample. Accordingly, the behavior of the material can be better characterized by normalizing the absorbed power with respect to the total power reaching the sample, that is, available power minus reflected power. The absorption coefficient, $\boldsymbol{C}_{\text {abs }}$, is then obtained as

$$
C_{\mathbf{a b s}}=\frac{1-\left|S_{11}\right|^{2}-\left|S_{21}\right|^{2}}{1-\left|S_{11}\right|^{2}} .
$$

The scattering parameters are measured by means of a HP 8720-B network analyzer. An RG-58 coaxial line with a polyethylene dielectric $\left(\varepsilon_{r}=2.28\right)$ is used as a sample holder, showing an attenuation of $0.76 \mathrm{~dB} / \mathrm{m}$ at $1 \mathrm{GHz}$. As the coaxial length of our setup is small (about $7 \mathrm{~cm}$ ), the attenuation due

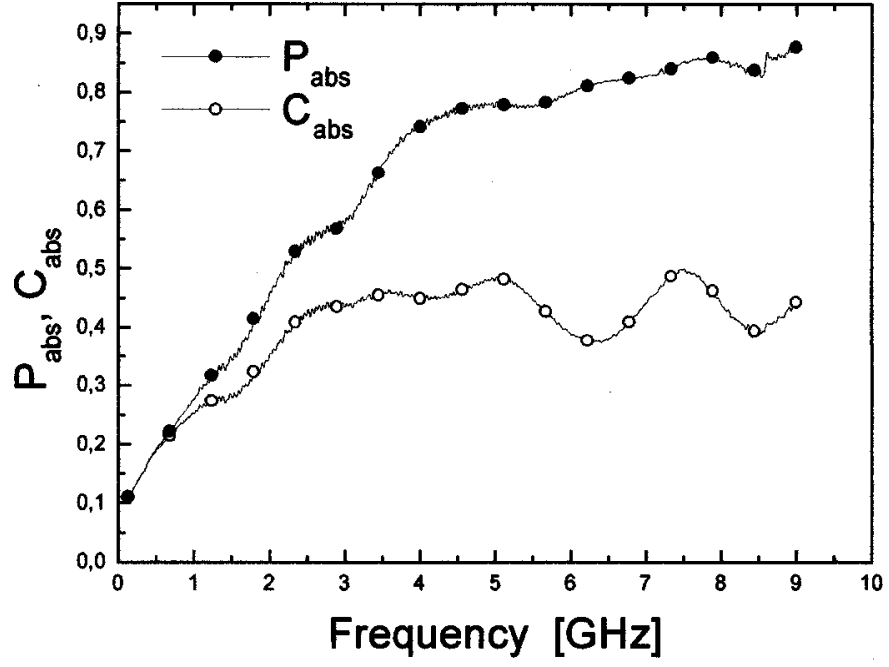

Fig. 3. Normalized absorbed power $\left(\boldsymbol{P}_{\mathbf{a b s}}\right)$ and absorption coefficient $\left(\boldsymbol{C}_{\mathbf{a b s}}\right)$ evolution from 0.15 up to $9 \mathrm{GHz}$ for a $\mathrm{Co}_{77.5} \mathrm{Si}_{13.5} \mathrm{~B}_{9}$ ribbon, $77 \mathrm{mg}$ in weight.

to the line $(0.05 \mathrm{~dB})$ is negligible compared to that due to the metallic glass in the frequency interval used which ranges from 0.15 up to $9 \mathrm{GHz}$.

\section{Resonance Frequency}

The analysis of the ferromagnetic resonance phenomena [17] gives information about intrinsic parameters of the material under study. The determination of the ferromagnetic resonance spectrum is achieved using a different arrangement of the samples. In this case, the coaxial line RG 402 has been used as a sample holder. The microwires are now placed parallel to the coaxial axis instead of being wound around the inner conductor. A short circuit is attached at one of the edges of the line, so that the absorption properties are fully determined by the $S_{11}$ parameter [see Fig. 1(b)]. The coaxial line is introduced into a solenoid which supplies a longitudinal (parallel to the axis of the sample) dc magnetic field in order to nearly saturate the magnetization of the sample and to excite the resonance. A perpendicular ac magnetic field of a transverse electromagnetic (TEM) propagating mode in the coaxial line provides the energy required for the precession of the magnetization. The resonance frequency is evaluated through the measurement of the scattering parameter $\boldsymbol{S}_{11}$ since the energy absorption reaches a maximum at the resonance.

\section{EXPERIMENTAL RESULTS AND ANALYSIS}

\section{A. Absorption Measurements}

The evolution of the normalized absorbed power as well as the absorption coefficient from 0.15 up to $9 \mathrm{GHz}$ for a $\mathrm{Co}_{77.5} \mathrm{Si}_{13.5} \mathrm{~B}_{9}$ ribbon (77 mg weight) are shown in Fig. 3 . The normalized absorbed power seemingly shows an oscillation for frequencies above $4 \mathrm{GHz}$. This oscillation may be attributed to the modification of the reflection properties of the sample holder, and not to intrinsic changes in the material. The absorption coefficient increases almost linearly with the frequency in the whole measured range and shows a change in the slope at around $4 \mathrm{GHz}$. 


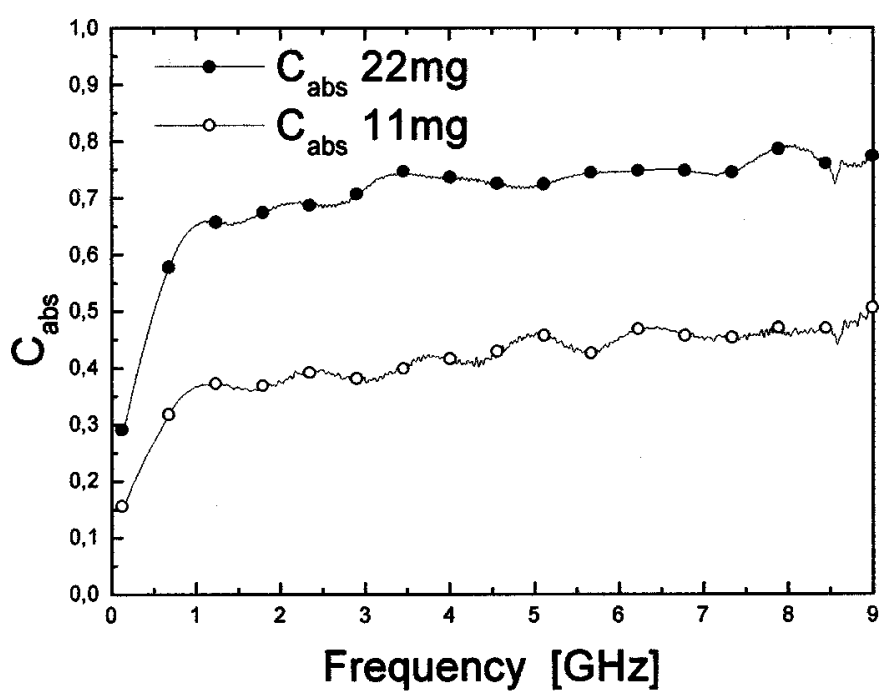

Fig. 4. Absorption coefficient evolution for $\mathrm{Co}_{68} \mathrm{~B}_{1.5} \mathrm{Si}_{10} \mathrm{Mn}_{7}$ microwires, $22 \mathrm{mg}$ in weight (solid circles), and for a sample which has been built by interweaving $11 \mathrm{mg}$ of such Co-rich microwires with $31 \mathrm{mg}$ of nylon (open circles).

Due to their tiny dimensions, samples in the shape of microwires are extremely difficult to handle. In order to overcome this problem and facilitate their manipulation, samples are usually prepared by interweaving the microwires under study with another material. Fig. 4 compares the absorption coefficient spectrum in the range from 0.15 to $9 \mathrm{GHz}$ for $\mathrm{Co}_{68} \mathrm{~B}_{15} \mathrm{Si}_{10} \mathrm{Mn}_{7}$ microwires having a weight of $22 \mathrm{mg}$ (solid circles) and for a sample which has been built by interweaving $11 \mathrm{mg}$ of such Co-rich microwires with $31 \mathrm{mg}$ of nylon (open circles). As can be concluded from Fig. 4, the nylon does not affect the behavior of the material in the microwave frequency range, as the general qualitative trend is the same in both cases. The absorption increases steeply until $1 \mathrm{GHz}$ and then remains nearly constant for higher frequencies. The qualitative discrepancy between both curves is easily ascribed to the difference in the weight of active magnetic material in both samples. The absorption coefficient is roughly proportional to the weight of magnetic material contained in the samples.

\section{B. Resonance Measurements}

According to previous experiments [18], [19], Co-rich microwires exhibit a magnetic domain structure characterized by a circumferential easy axis arising from the distribution of the magnetoelastic anisotropy. That forces the magnetization to lie along the surface of the wire perpendicular to its axis. This circumferential magnetization near the surface of the wire is strongly affected by the circular and oscillating magnetic field of the TEM mode which propagates along the coaxial line.

Consequently, unrolling the outer shell into a plane results in a configuration similar to that of a film with an in-plane magnetic anisotropy which is perpendicular to the in-plane static magnetic field [20]. The following coordinate system is considered: the dc magnetic field is applied along the $z$ axis, while the $y$ axis is taken as the easy direction for the magnetization, and the $x$ axis is perpendicular to the thin-film surface. Considering both the demagnetizing effects and the magnetic anisotropy ac-

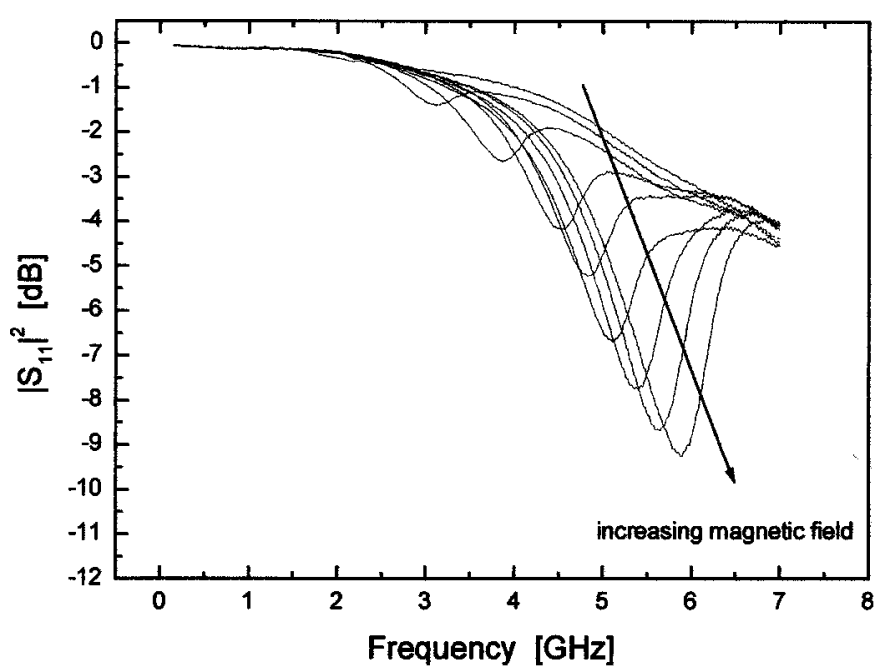

Fig. 5. Evolution of the square of the scattering parameter with the frequency for different values of the increasing applied field up to $700 \mathrm{Oe}$.

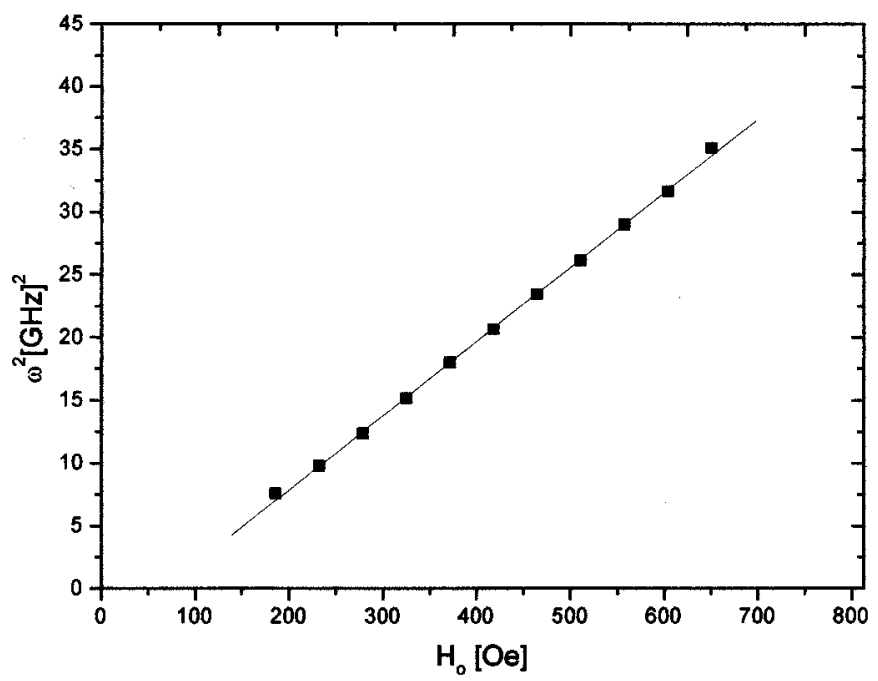

Fig. 6. Dependence of the second power of the ferromagnetic resonance frequency on the static applied field.

cording to the notation given by Craik [21], the demagnetizing factors are $D_{x}=1, D_{z}=D_{y}=0$, and the anisotropy factors are given by $D_{x}^{k}=D_{z}^{k}=0, D_{y}^{k}=-2 K / \mu_{o} M_{s}^{2}, K$ being the uniaxial anisotropy energy, $\mu_{o}$ the free-space magnetic permeability, and $\boldsymbol{M}_{\boldsymbol{s}}$ the saturation magnetization. Consequently, the resonance frequency can be written as

$$
\omega=\gamma \mu_{0} \sqrt{\left(H_{0}+M_{s}\right)\left(H_{0}-\frac{2 K}{\mu_{0} M_{s}}\right)}
$$

$\boldsymbol{H}_{\mathbf{0}}$ being the dc applied field and $\boldsymbol{\gamma}$ the gyromagnetic constant. As the saturation magnetization is much larger than the maximum applied field $\left(\boldsymbol{M}_{\boldsymbol{s}} \gg \boldsymbol{H}_{\mathbf{0}}\right)$, the resonance frequency can be approximated as follows:

$$
\omega=\gamma \mu_{0} \sqrt{M_{s}\left(H_{0}-H_{k}\right)}
$$

where $\boldsymbol{H}_{\boldsymbol{k}}=\mathbf{2 K} / \boldsymbol{\mu}_{\mathbf{0}} \boldsymbol{M}_{\boldsymbol{s}}$ is the transverse anisotropy field.

Fig. 5 shows the evolution of $\left|S_{11}\right|^{2}$ with the frequency for increasing values of the static applied field. The minimum of each curve corresponds to the resonance frequency for a given field. 


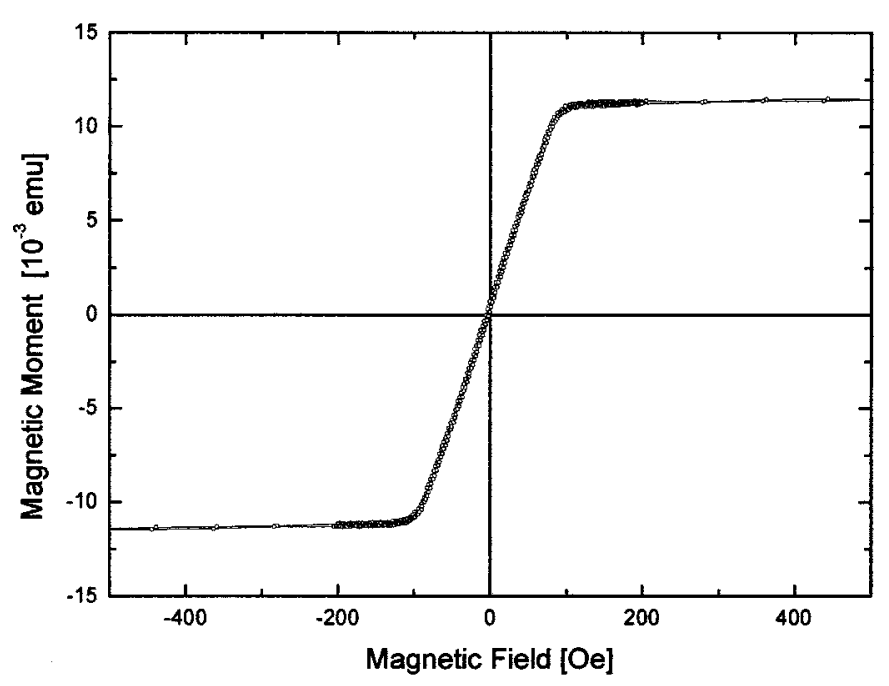

Fig. 7. Hysteresis loop of $\mathrm{Co}_{68} \mathrm{~B}_{15} \mathrm{Si}_{10} \mathrm{Mn}_{7}$ microwires measured using a vibrating sample magnetometer.

As can be deduced from (4), the second power of the resonance frequency increases linearly with the applied field $H_{0}$.

Fig. 6 shows the experimental data of the squared resonance frequency as a function of the applied field, and their fitting to a linear behavior. The anisotropy field obtained from the extrapolation of the fitting down to $\boldsymbol{\omega}=0$ is $\boldsymbol{H}_{\boldsymbol{k}}=68 \mathrm{Oe}$. In order to check the validity of this technique to obtain information on the magnetic anisotropy, the longitudinal hysteresis loop was obtained using VSM. From the loop (see Fig. 7) a transverse anisotropy field of 80 Oe can be easily deduced, which agrees reasonably with that obtained by the resonance technique.

\section{CONCLUSION}

In summary, an alternative method has been successfully employed to determine the power absorption in the microwave range of Co-rich metallic glasses in the shape of ribbons and microwires.

A coaxial line is modified by the substitution of the dielectric by the ferromagnetic sample. The change in the scattering parameters produced depends on the properties of the ferromagnetic sample and its arrangement in the coaxial line. This has allowed us to determine the absorption properties of the studied samples. When the microwires are parallel to the coaxial axis and a de magnetic field is applied along this direction, the ferromagnetic resonance frequency can also be found. Furthermore, the anisotropy field of the microwires has been inferred from the linear increase of the second power of the ferromagnetic resonance frequency with the applied field, and the consequent value fits reasonably with that deduced from the low-frequency hysteresis loop.

\section{ACKNOWLEDGMENT}

The authors would like to express their appreciation to J. M. Catalá, C. Devece, and D. Sánchez for their help.

\section{REFERENCES}

[1] A. Antonov, A. Granovski, A. Lagar'kov, N. Perov, N. Usov, and T. Furmanova, "The features of GMI effect in amorphous wires at microwaves," Physica A, vol. 241, pp. 420-424, 1997.

[2] P. M. Jacquart and O. Acher, "Permeability measurement on composites made of oriented metallic wires from 0.1 to $18 \mathrm{GHz}$," IEEE Trans. Microwave Theory Tech., vol. 44, pp. 2116-2120, Nov. 1996.

[3] L. V. Panina, K. Mohri, T. Uchiyama, and M. Noda, "Giant magnetoimpedance in Co-rich amorphous wires and films," IEEE Trans. Magn., vol. 31, pp. 1244-1260, Mar. 1995.

[4] D. Ménard, M. Britel, P. Ciureanu, and A. Yelon, "Giant magnetoimpedance in a cylindrical magnetic conductor," J. Appl. Phys., vol. 84, no. 5, pp. 2805-2814, 1998.

[5] S. E. Lofland, S. M. Bhagat, M. Domínguez, J. M. García-Beneytez, F. Guerrero, and M. Vázquez, "Low field microwave magnetoimpedance in amorphous microwires," J. Appl. Phys., vol. 85, pp. 4442-4444, 1999.

[6] O. Ishii, M. Senda, K. Takei, Y. Koshimoto, and T. Mori, "Application of Co-based amorphous ribbon to a noise filter and a shielded cable," $J$. Appl. Phys., vol. 79, no. 8, pp. 5483-5485, 1996.

[7] A. N. Medina, M. Knobel, S. Salem-Sugui, and F. G. Gandra, "Resonant microwave cavity response of amorphous ribbons," J. Appl. Phys., vol. 79, no. 8, pp. 5462-5464, 1996.

[8] S. Lefrançois, D. Pasquet, and G. Mazé-Merceur, "A new model for microwave characterization of composite materials in guided-wave medium," IEEE Trans. Microwave Theory Tech., vol. 44, pp. 1557-1562, Sept. 1996.

[9] D. K. Ghodgaonkar, V. V. Varadan, and V. K. Varadan, "Free-space measurement of complex permittivity and complex permeability of magnetic materials at microwave frequencies," IEEE Trans. Instrum. Meas., vol. 39, pp. 387-394, Apr. 1990.

[10] P. Queffelec, P. Gelin, J. Gieraltowski, and J. Loáec, “A microstrip device for the broad band simultaneous measurement of complex permeability and permittivity," IEEE Trans. Magn., vol. 30, pp. 224-230, Mar. 1994.

[11] N.-E. Belhadj-Tahar, A. Fourrier-Lamer, and H. de Chanterac, "Broad-band simultaneous measurement of complex permittivity and permeability using a coaxial discontinuity," IEEE Trans. Microwave Theory Tech., vol. 38, pp. 1-7, Jan. 1990.

[12] J. M. García-Beneytez, F. Vinai, L. Brunetti, H. García-Miquel, and M. Vázquez, "Study of magneto impedance effect in the microwave frequency range for soft magnetic wires and microwires," Sens. Actuators A, Phys., vol. 81, pp. 78-81, 2000.

[13] O. Acher, P. M. Jacquart, J. M. Fontaine, P. Baclet, and G. Perrin, "High impedance anisotropic composites manufactured from ferromagnetic thin films for microwave applications," IEEE Trans. Magn., vol. 30, pp. 4533-4535, Nov. 1994.

[14] M. Vázquez and A. P. Zhukov, "Magnetic properties of glass-coated amorphous and nanocrystalline materials," J. Magn. Magn. Mater, vol. 160, no. 1, pp. 223-228, 1996.

[15] H. Chiriac, T. A. Ovari, and Gh. Pop, "Internal stress distribution in glass-covered amorphous magnetic wires," Phys. Rev. B, Condens. Matter, vol. 52, no. 14, pp. 10104-10113, 1995.

[16] O. Acher, P. Le Gourriérec, G. Perrin, P. Baclet, and O. Roblin, "Demonstration of anisotropic composites with tuneable microwave permeability manufactured from ferromagnetic thin films," IEEE Trans. Microwave Theory Tech., vol. 44, pp. 674-684, May 1996.

[17] C. Kittel, "On the theory of ferromagnetic resonance absorption," Phys. Rev., vol. 73, no. 2, pp. 155-161, 1948.

[18] H. Chiriac, G. Pop, T.-A. Ovari, and F. Barariu, "Magnetic behavior of negative and nearly zero magnetostrictive glass-covered amorphous wires," IEEE Trans. Magn., vol. 32, pp. 4872-4874, Sept. 1996.

[19] J. Velazquez, M. Vazquez, and A. P. Zhukov, "Magnetoelastic anisotropy distribution in glass-coated microwires," J. Mater. Res., vol. 11, no. 10, pp. 2499-2505, 1996.

[20] P. Ciureanu, M. Britel, D. Ménard, A. Yelon, C. Akyel, M. Rouabhi, R. W. Cochrane, P. Rudkowski, and J. O. Strom-Olsen, "High frequency behavior of soft magnetic wires using the giant magnetoimpedance effect," J. Appl. Phys., vol. 83, no. 11, pp. 6563-6565, 1998.

[21] D. Craik, Magnetism: Principles and Applications. Chichester, U.K.: Wiley, 1995. 